Assurances et gestion des risques

Insurance and Risk Management

\title{
Le contrat d'assurance face à l'aléa : mythe ou réalité ?
}

\section{Nguema-Evié Thomas}

Volume 85, numéro 1-2, juin 2018

URI : https://id.erudit.org/iderudit/1051318ar

DOI : https://doi.org/10.7202/1051318ar

Aller au sommaire du numéro

Éditeur(s)

Faculté des sciences de l'administration, Université Laval

ISSN

1705-7299 (imprimé)

2371-4913 (numérique)

Découvrir la revue

Citer ce document

Thomas, N.-E. (2018). Le contrat d'assurance face à l'aléa : mythe ou réalité ? Assurances et gestion des risques / Insurance and Risk Management, 85(1-2), 119-130. https://doi.org/10.7202/1051318ar

Tous droits réservés $\odot$ Faculté des sciences de l'administration, Université Laval, 2018
Ce document est protégé par la loi sur le droit d'auteur. L'utilisation des services d'Érudit (y compris la reproduction) est assujettie à sa politique d'utilisation que vous pouvez consulter en ligne.

https://apropos.erudit.org/fr/usagers/politique-dutilisation/ 


\section{LE CONTRAT D'ASSURANCE FACE À L'ALÉA: MYTHE OU RÉALITÉ?}

\section{Monsieur Nguema-Evié Thomas}

L'approche historique de l'évolution du contrat d'assurance réfère à l'aléa dans les différentes étapes de sa formation ainsi que dans la détermination de la prime exigée en contrepartie de la prestation de services délivrés par l'assureur.

En effet, la génèse relativement controversée de l'assurance, lui conférerait une origine antique la faisant remonter à la pratique du prêt à la grosse aventure ${ }^{1}$ connu encore sous l'appellation de Nauticum foenus.

L'aléa, critère déterminant dans l'observation des obligations, semble coordonner le concept de mutualité dans une approche scientifique du contrat ${ }^{2}$, et pose en toile de fond la question de la nature même du contrat d'assurance ${ }^{3}$.

À cet effet, ,l'usage de la notion d'aléa comme outil d'appréciation des obligations du contrat nous invite à une analyse de son emploi tant sur le plan technique que sur celui employé par les juridictions compétentes de cet instrument en faveur de la recherche d'un équilibre contractuel.

Pour ce faire, un éclaircissement sémantique de la notion d'aléa ${ }^{4}$ impose qu'on le présente sous ces deux aspects principaux:

Sur le plan technique, il faut rappeler avant tout que la question de l'aléa, selon Claude Fluet, ne fait pas abstraction de sa dimension morale. Il avance au sujet des contrats d'assurance responsabilité que "Les effets démotivants de l'assurance sont bien connus. Dans la mesure où elle protège contre les conséquences pécuniaires de poursuites en dommages-intérêts, l'assurance de responsabilité semble devoir par le fait même affaiblir les incitations à la prudence "s. En d'autres termes 
l'aléa moral est "une relation "principal" - "agent" " ou "le principal fait face à de l'aléa moral lorsque l'agent peut prendre des décisions "non observables". Ces décisions, du fait de la divergence d'objectifs entre ces deux protagonistes, peuvent ne pas être dans l'intérêt du principal. L'assurance maladie est un exemple d'aléa moral dans la mesure où les dépenses résultant de la transaction entre le patient et le médecin ne peuvent être contrôlées par l'assureur "6 Ainsi, techniquement, l'aléa moral ne serait rien d'autre que l'absence de prédiction du comportement de l'assuré par des lois statistiques et probalistiques qui, à travers son comportement, participerait à l'aggravation du risque.

Sur le plan juridique, l'aléa du contrat d'assurance se définit à travers l'ensemble de ces caractères, à savoir l'incertitude de l'évènement qui le constitue d'une part et de l'égalité informationnelle des parties face à cet évènement d'autre part. Dans leur ouvrage sur les contrats spéciaux, O.FARDOUX et J-C.PLANQUE définissent le contrat aléatoire comme étant un contrat "qui repose sur le hasard qui provoquera un gain ou une perte" 7 selon ces derniers, "la particularité du contrat aléatoire est que la prestation de l'une des parties n'a pas pour équivalent une chose ou une prestation ${ }^{8}$. Cette position est également partagée par le doyen Didier LLULLES dans son ouvrage Précis des assurances terrestres au Québec où il affirme que "entre l'assureur et le preneur, "il y a chance de gain ou de perte pour les deux parties" " faisant reposer l'aléa sur la notion de chance voire de hasard.

Cependant, que l'on pense à la chance ou hasard comme fondement de la nature du contrat d'assurance, il renvoie systématiquement à la signification de ces différentes notions dans un souci de scientificité et de logique de nos réflexions.

Ainsi, la chance, selon Gaétan Saint-Pierre, dans son étude étymologique "vient du latin populaire cadentia, participe présent substantivé de cadere "tomber, se terminer". Lequel renvoie, selon G. Saint-pierre, plus concrètement "au jeu, à la manière dont tombent les dés ou, si l'on préfêre, à la manière dont "se termine" leur course. ${ }^{10 "}$

L'étude s'approfondit par l'explication d'une notion à partir de laquelle s'opère un rapprochement avec celle de hasard. G. Saint-Pierre précise à ce propos que "À l'origine, le mot chance signifie donc "hasard" ou "manière dont un événement se produit; et, à l'instar de heur, par exemple, la chance sera qualifiée de bonne (bonne chance) ou de mauvaise (mal chance) selon le cas." 
G. Saint-Pierre précise en plus que "Dès le Moyen Âge, le mot prend généralement, au singulier, le sens de "beureux hasard", de "sort favorable": avoir son jour de chance. On lui oppose alors la malchance (malchance, XIII'), terme désignant originellement le fait de mal tomber. Les dérivés chanceux (début XVII') et, surtout, malchanceux (vers 1875) sont beaucoup plus récents. „11

À la lumière de ces concepts, ressort une nécessité évidente de rapprochement entre le contrat d'assurance et le régime juridique des contrats de jeu ou de pari posé aux articles 1965 et suivants du code Civil Français et 2629 et suivants du Chapitre XVI du livre V du code civil du Québec. Comme lees articles disposstipulent ces articles, « $L a$ loi n'accorde aucune action pour une dette du jeu ou pour le paiement d'un pari ${ }^{12}$ et "Les contrats de jeu et de pari sont valables dans les cas expressément autorisés par la loi.

Ils le sont aussi lorsqu'ils portent sur des exercices et des jeux licites qui tiennent à la seule adresse des parties ou à l'exercice de leur corps, à moins que la somme en jeu ne soit excessive, compte tenu des circonstances, ainsi que de l'état et des facultés des parties. " ${ }^{13}$ Le code Civil du Québec rappelle cette proximité entre la chance ou l'idée du jeu qui y est conceptualisée et le régime juridique applicable à l'activité d'assurance tel qu'il est mentionné au Règlement d'application de la Loi sur les assurances (chapitre A-32, a. 420 et 420.1) en son article 2 lequel dispose que: "La demande de constitution d'une compagnie d'assurance doit également être accompagnée d'un plan d'affaires comprenant les états financiers d'ouverture, les prévisions financières quinquennales et une description de la structure organisationnelle. Ce plan d'affaires est appuyé d'une projection actuarielle, sur une période d'au moins 5 ans, du bilan, de l'état des résultats, de l'état des bénéfices non répartis et de la suffisance de capital. La projection actuarielle contient une description des hypothèses de calcul retenues. Elle est établie par un actuaire membre (fellow) de l'Institut canadien des actuaires exerçant sa profession en assurance de personnes ou en assurance de dommages, selon les activités que la compagnie d'assurance se propose d'exercer."

L'actuariat étant une science visant à modéliser le hasard ou la chance afin de lui faire perdre ses caractères d'imprévisibilité en vue de la rendre prédictible, on pourrait dès lors s'interroger sur la formulation conventionnelle du contrat d'assurance. En utilisant l'aléa comme critérium à l'instar de l'article 1382 du code Civil du Québec lequel dispose qu' Il est aléatoire lorsque l'étendue de l'obligation ou des 
avantages est incertaine." ce qui, d'une certaine façon, justifierait le principe de non restitution de la prime versée en cas d'année écoulé in bonis.

Cependant, il est important de rappeler que si les notions de chance et de hasard datant de l'antiquité et revisitées durant le moyen âge ont fortement influencé le régime du contrat aléatoire du code Civil français de 1804, les révolutions opérées par Blaise Pascal sur la rationalisation du hasard à travers la promotion des calculs liés aux probabilités auraient dû soulever la question de catégorisation du contrat d'assurance dans la sphère des contrats aléatoires dès l'édiction du code Civil.

L'assimilation du contrat d'assurance à un contrat aléatoire inspiré du code Civil français, soulève la question des caractéristiques du risque couvert par le contrat.

Ce risque est-il un évènement contrôlable dont seule la survenance exceptionnelle serait garantie par un contrat sui generis de prestation de service assortie d'une condition suspensive assimilé à un cautionnement social ou, au contraire, l'évènement demeurerait sous l'influence du hasard ou de la chance, non maitrisable et serait ainsi un contrat de pari à part entière sur l'avenir?

Cette question soulève l'interrogation relative à la validité des critères usuels du contrat d'assurance par les professionnels du droit et de l'industrie de l'assurance.

Peut-on encore parler de contrat aléatoire au sujet du contrat d'assurance lorsque ce dernier repose sur une rationalité du risque?

L'exemple du recours à de nouvelles technologies de l'informatique et de la communication (NTIC) à l'instar des big data marquant le passage du web social à un web prédictif, le débat de l'accès à l'information relativement aux données médicales pour une optimisation de la sélection du risque soulèvent autant de questions sur la validité de l'usage du critère aléatoire qu'elles invitent à un approfondissement rigoureux de ces questionnements.

Ainsi, afin de fournir quelques éléments de réponse à cette question, nous aborderons dans un premier temps, l'analyse de l'aléa dans le contentieux d'assurance en France et au Québec (I) avant d'établir l'obsolescence du critère d'aléa comme élément d'appréciation du contrat d'assurance (II) 


\section{ANALYSE dE L'ALÉA DANS LE CONTENTIEUX D'ASSURANCE: L'EXEMPLE DU DROIT FRANÇAIS}

L'analyse du contentieux d'assurance tant en droit Français qu'en droit Québécois présente des situations particulières d'appréciation du critère aléatoire. De plus, pour des raisons méthodologiques, notre orientation se basera sur l'exemple du régime des nullités du droit des contrats Français lequel présente plusieurs points communs avec le droit Québec en matière d'assurance.

À cet effet, notre réflexion reposera sur la question de l'appréciation du critère "aléa» d'un point de vue légal (A) d'une part, avant de l'aborder d'un point de vue doctrinal d'autre part (B)

\section{A. L'appréciation de l'aléa par la loi: vers une critique du texte.}

L'étude de l'aléa en matière de contrat d'assurance présente un intérêt majeur d'appréciation du régime des nullités du contrat. En effet, suite aux diverses polémiques ayant subsistées, il est fondamental de poser brièvement les contours légaux ayant soulevé la controverse et constituant, dans une certaine mesure, l'objet de la présente étude.

À la lecture de l'article 1964 du code Civil, apparait l'affirmation selon laquelle "le contrat aléatoire est une convention réciproque dont les effets, quant aux avantages et aux pertes, soit pour toutes les parties, soit pour l'une ou plusieurs d'entre elles, dépendent d'un événement incertain. Tels sont: le contrat d'assurance, le jeu et le pari, le contrat de rente viagère. „14

Cette position législative propose un certain nombre d'éclaircissements quant à la mise en perspective de l'aléa tel que soulevé précédemment. L'analyse économique du droit à travers l'ouvrage d'Ejan MACKAAY ${ }^{15}$ a apporté quelques précisions notables ainsi que les travaux de Frank Knight qui soulevait déjà la nécessité de dissocier le risque de l'incertitude.

Selon ce dernier, le risque s'apparentait aux "phénomènes" dont on avait au moins "une connaissance statistique» tandis que "l'incertitude» était employée indistinctement pour parler des aléas dont la "connaissance» n'était pas disponible. 
Il ressort de cette distinction, pour le moins pertinente, des observations majeures sur l'adjonction des concepts de risque et d'aléas au sein de la théorie du contrat d'assurance: les éléments constitutifs du contrat d'assurance reposant sur la théorie générale des contrats, à savoir, l'objet, la capacité, le consentement et la cause et ce, jusqu'à la réforme 2016-131 du 10 février 2016. Conservant après celle-ci, le consentement des partis, leur capacité de contracter, un contenu licite et des conditions particulières à savoir le risque aléatoire, la prime et la prestation de garantie de l'assureur uniquement. S'ensuit la question du paradoxe des critères particuliers du contrat d'assurance comme outils de son appréciation par les tribunaux.

Le risque (minimisé par les calculs actuariels?) ayant pour conséquence rationnelle de faire perdre au contrat d'assurance sa nature aléatoire et l'aléa lui conférant un régime juridique identique aux paris ou aux jeux.

Ces réflexions so0nt confortées par la position de la jurisprudence, qui à travers sa décision de 1999 pose la question de la place de l'aléa dans l'appréciation du contrat d'assurance.

En effet, l'étude de la jurisprudence, sur la question de la nullité d'un contrat d'assurance pour défaut d'aléa assimilé à la cause, a contribué à relancer le débat sur la possibilité de conclure des contrats d'assurance sans aléa, voire même de conférer à cette notion une place secondaire dans l'appréciation du contrat d'assurance dépendamment des intérêts en cause. La question sous-jacente étant la détermination de la méthode d'évaluation du critère de priorité ou de finalité à partir duquel apprécier «l'aléa» ou le "risque» dans le contrat d'assurance?

À titre d'illustration, dans une décision de Cass. 1re civ., 9 novembre 1999; SA Groupement Français d'Assurances (GFA) et a. c/ Cie Auxiliaire et a. la cour de cassation a affirmé que "attendu que la nullité du contrat d'assurance pour absence d'aléa est une nullité relative qui ne peut être invoquée que par celui dont la loi qui a été méconnue tendait à assurer la protection; que c'est donc à bon droit, que la cour d'appel, après avoir relevé que le défaut d'aléa n'entraînait pas une nullité absolue et que l'action en nullité, qui avait pour objet la clause d'effet rétroactif stipulée au contrat d'assurance n'appartenait pas aux sociétés qui entendaient s'en prévaloir, a décidé que l'assureur, subrogé dans les droits de son assuré, était recevable à agir contre ces sociétés; que le moyen n'est donc pas fondé; Par ces motifs:-Casse et annule, mais seulement en ce qu'il a dit que l'assureur était recevable à $\operatorname{agir}^{16}$ » 
Cette décision novatrice est intéressante dans la mesure où elle rattache l'action en nullité pour défaut d'aléa à une atteinte de l'intérêt d'ordre privé et non public la faisant relever de facto au régime de la nullité relative. Les exigences de l'ordre public poursuivies par les conditions de validité du contrat à observer ne semblent plus considérer l'aléa comme un critère essentiel à la validité du contrat.

Une tentative d'explication fuût apportée par la doctrine, laquelle mérite d'être abordée à la suite de ces propos.

\section{B. L'appréciation de l'Aléa par la doctrine}

Sur le plan doctrinal la position du professeur Groutel ne manque pas de susciter un intérêt. Ce dernier évoque l'opposition d'appréciation du régime des nullités entre les classiques et les modernes, laquelle semble aboutir à une instrumentalisation des éléments constitutifs du contrat d'assurance.

En effet, pour les classiques la cause devant être assortie d'une nullité absolue est assimilée à l'aléa dans les contrats aléatoires laquelle affecte le contrat d'assurance d'une nullité absolue en cas d'absence de celui-ci. Tandis que pour les modernes, la nature de la nullité de la cause est appréciée en regard des intérêts à protéger.

Groutel soulève à cet effet que "La Cour choisit ainsi clairement d'appliquer la théorie moderne des nullités plutôt que la théorie classique attachant la nature de la nullité à l'état du contrat. En effet, selon la doctrine moderne dominante, la nature de la nullité attachée au non-respect d'un texte dépend de la vocation de celui-ci à protéger ou non un intérêt général. Certes, la frontière entre l'intérêt particulier et l'intérêt général est difficile à tracer puisqu'en l'absence de tout intérêt général, le législateur se serait abstenu d'édicter une règle d'ordre public telle l'article L. 121-15 du Code des assurances."

Ce texte posant comme plausible la possibilité de contracter des obligations d'assurances dont l'exigence d'observation de l'essence de celui-ci, à savoir l'aléa, ne relèverait pas de l'ordre public mais de la précaution des parties.

La question de la preuve se posant par ailleurs, il en ressort une prédilection en faveur d'une interprétation utilitariste du contrat au détriment d'une justice commutative. 
Par ailleurs, la tentative d'esquiver le débat par la suppression textuelle de la cause par la réforme des obligations de 2016 ne purge pas l'ensemble des questionnements afférents.

L'un des arguments principaux fut justement la difficulté à établir des critères précis de la notion de cause. Ce qui avait pour conséquence de rendre inattractif le marché Français. Au-delà de l'intérêt général de la réforme pour la théorie des contrats, la question d'appréciation du contrat d'assurance dans ses éléments demeure toujours pertinente.

\section{L'OBSOLESCENCE DE LA NOTION D'ALÉA}

Le point de vue à portée juridique partagé ici s'articule autour de l'appréciation juridique de l'obsolescence de l'aléa (B) laquelle découle de l'obsolescence historico-scientifique (A).

\section{A. L'obsolescence historico-scientifique.}

L'étude historique du droit des contrats modernes dans leur relation avec l'aléa montre à quel point son emploi est sans intérêt dans la pratique des contrats d'assurance.

En effet, si la renaissance a contribué à la découverte de nouvelles méthodes visant à rationaliser le réel, par exemple avec les probabilités de Pascal, il appert qu'elle a aussi permis de faire perdre au hasard certaines de ses vertus dans des situations précises posées par la statistique.

Ainsi, l'apparition du code Civil Français de 1804 marque la première limite des justesses des catégories juridiques prévues par celui-ci.

Les raisons sont nombreuses. Trois d'entre elles peuvent recouper l'essentiel de cette position. Tout d'abord, sur le plan juridique, la pratique de l'assurance n'a pas eu la même importance en Angleterre qu'en France par le législateur.

La raison principale repose sur la dynamique des révolutions industrielles, ce qui explique qu'il ait fallu attendre le XVII ${ }^{\mathrm{e}}$ siècle pour voir les premières institutions d'assurances? au sein du royaume, 
notamment grâce à Colbert en 1668, pour encadrer "l'aléa" maritime. La première loi en matière d'assurance de dommage résultant de la loi du 13 juillet 1930 soit au xx siècle, marqua le début de la reconnaissance par l'Etat du risque dommage IARD.

En plus des raisons juridiques, figurent des raisons culturelles et politiques. eEn effet, l'assurance a toujours été perçue comme un pari sur la vie ou sur la durée des choses. Cette conception entrant en contradiction avec les mours était renforcée ete jugée comme immorale et avide. À cet effet, Portalis a pu dire au sujet des assurances vies que "l'homme est hors de prix: sa vie ne saurait être un objet de commerce, sa mort ne peut devenir la matière d'une spéculation mercantile.». ${ }^{17}$ Cette position fut partagée également par l'Église qui jugeait comme attentatoire à l'ordre divin la spéculation sur la vie humaine. Pour le révérend Claude de la Combière en 1697, "tous les bommes sont si persuadés de l'incertitude de la mort qu'ils ne voudraient pas hasarder une partie de leurs biens sur l'espérance d'une longue vie, tant ils croient cette espérance mal fondée».18

Enfin, sur le plan économique, la spéculation sur la vie ou sur la durée des choses était considérée comme non productive de richesse. La théorie des physiocrates a confirmé l'importance de l'immobilier comme facteur de richesse si bien que toutes formes de production mobilière étaient jugées comme futiles.

Des adages juridiques encore usités de nos jours en témoignent, à savoir Res mobilis res vilis res immobilis res nobilis ont confirmé cette place secondaire dans laquelle était assimilée le contrat d'assurance.

Mais l'obsolescence de l'aléa est aussi à observer sur le plan juridique.

\section{B. L'obsolescence juridique de l'aléa}

La dimension juridique de l'obsolescence de l'aléa s'appréciera, selon notre approche, sous l'angle du positivisme normativiste incarné par Hans Kelsen.

En effet, selon cette approche nous considérerons le droit des assurances dans sa nature intrinsèque à savoir un ensemble coordonné et rationnel de normes visant à encadrer l'activité d'assurance tant dans sa dimension productive que dans sa dimension consommatrice. 
À cet égard et en dépit de l'importance des informations en cause, nous procéderons à une circonscription de notre étude pour nous intéresser à l'approche consommatrice de l'assurance, sans perdre de vue la nécessité de jonction de l'ensemble de la matière à travers le concept d'aléa.

En effet, si selon l'approche positiviste le contrat est valide du fait de sa conformité à la loi, cela soulève la question de savoir quelles sont les caractéristiques des éléments du contrat d'assurance à partir duquel celui-ci fonde sa validité au regard de la loi?

La question ainsi posée nous amène à tenir compte des spécificités du contrat d'assurance lequel repose d'une part sur la théorie générale des contrats et d'autre part sur une théorie spéciale propre au contrat d'assurance.

Si la théorie générale diffère d'un système à un autre en fonction des objectifs du législateur comme le démontre la récente réforme des obligations en France par l'ordonnance 2016-131 de février 2016, la théorie spéciale du contrat d'assurance semble relever d'un ordre intégré aux sociétés développées qui utilisent une approche commune d'appréciation du contrat d'assurance.

Ainsi, la nécessité du risque de l'aléa et des obligations des parties au contrat d'assurance dont notamment le paiement de la prime pour l'assuré et la prestation de couverture souscrite par ce dernier pour l'assureur constituent des éléments considérés comme fondamentaux à la validité du contrat d'assurance.

Bien entendu sur le plan technique, d'autres critères peuvent être retenus à savoir la dispersion du risque, la sélection de celui-ci ainsi que la constitution d'une mutualité comme éléments principaux de constitution technique d'un contrat d'assurance.

Aussi, adopterons-nous la dimension juridique de la théorie spéciale du contrat d'assurance pour l'étude du concept d'aléa. En effet, s'il est établi que l'aléa est un hasard/chance il est à préciser avant tout que l'aléa n'est pas défini positivement par le législateur ${ }^{19}$ tant en droit Français qu'en droit Québécois.

La raison principale relevant du fait que le concept en soi n'est pas de nature juridique mais socio-culturel portant, en lui, l'idée d'absence d'information. Cette absence d'information, à distinguer scrupuleusement 
de la carence ou du concept de rétention, ne soulève pas tant la question de la cause ou de l'objet du contrat d'assurance mais de l'encadrement normatif auquel celui-ci est soumis.

Le régime général de type commutatif du contrat s'opposant ainsi au régime spécial de type aléatoire du contrat d'assurance. Mais plus encore, admettre l'aléa dans l'appréciation du contrat d'assurance serait le considérer comme un contrat dénué de connaissance d'information tant sur l'objet que sur la cause du contrat. Si bien que dans l'optique ou nous souhaiterions apprécier le contenu du contrat dans son objet ou sa cause tant objective que subjective seule la référence au hasard mériterait d'être retenue.

Il en ressort que, comparativement à l'aléa, seul le risque propre à l'incertitude mériterait d'être retenu comme élément d'appréciation du contrat d'assurance au coté des obligations des parties et des conditions techniques. Bien entendu, il en revient aux législateurs des divers états de se pencher sur la question afin d'améliorer le régime des contrats d'assurances.

\section{NOTES}

1. Marly, Pierre-Grégoire. Droit des assurances. Paris: Dalloz, 2013. p. 2-7

2. Petitclerc, Martin. La solidarité face au marché quelques réflexions sur l'histoire de la mutualité au Québec, revue international de l'économie sociale, $n^{\circ} 283$

3. André Bélanger and Joëlle Manekeng Tawali, Le spectre de la mutualité dans le contrat d'assurance. Revue générale de droit 392 (2009) : 297-328.

4. Aléa était le surnom sous lequel Minerve était révérée à Aléa en Arcadie, Étymologiquement, le terme d'aléa provient du latin alea, ae : coup de dés 3Ph. MALAURIE, L. AYNES, P.-Y. GAUTIER, Les contrats spéciaux, 3ème éd., Defrénois, 2007, n 972 4V. LASSERRE-KIESOW, «L'aléa», JCP éd. G, 2009.I.50 5M. VAN DE KERCHOVE et F. OST, Le droit ou les paradoxes du jeu, PUF, 1992, p. 1166 Art. 1964 du Code civil: "Le contrat aléatoire est une convention réciproque dont les effets quant aux avantages et aux pertes, soit pour toutes les parties, soit pour l'une ou plusieurs d'entre elles, dépendent d'un événement incertain.

5. Claude Fluet, «Assurance de responsabilité et aléa moral dans les régimes de responsabilité objective et pour faute», Revue d'économie politique 2002/6 (Vol. 112), p. 845-861.

6. Définition du glossaire du centre international de la recherche scientifique. http://www2.cnrs.fr/sites/communique/fichier/09_glossaire.pdf

7. Fardoux, Olivier, et Jean-Claude Planque. Contrats spéciaux. Paris : Bréal, 2011. p.166

8. Fardoux, Olivier, et Jean-Claude Planque. Contrats spéciaux. Paris: Bréal, 2011. p.166

9. Lluelles, Didier. Précis des assurances terrestres. 5e éd. Montréal : Éditions Thémis, 2009. p.25

10. Saint-Pierre, Gaétan. Etymologie, Volume 18, numéro 1, 2012.

11. Saint-Pierre, Gaétan. Etymologie, Volume 18, numéro 1, 2012.

12. Article 1965 du Code Civil Français

13. Article 2629 du code Civil du Québec

14. Article 1964 du code Civil 
15. Mackaay, Ejan. L'analyse economique du droit. Montréal, Québec: Bruxelles: Editions Thémis; Bruylant, 2000

16. Cass. 1re civ., 9 novembre 1999; SA Groupement Français d'Assurances (GFA) et a. c/ Cie Auxiliaire et a. (arrêt $\left.n^{\circ} 1706 P+B\right)$ [Juris-Data $n^{\circ} 003918$

17. Jean-Etienne-Marie Portalis, Discours, rapports et travaux inédits sur le Code civil, Paris Joubert Libraire de la cour de cassation, 1844 .

18. Claude de la Combière, Sermons Prêches: Devant Son Altesse Royale Madame La Duchesse D'York., volume 6.

19. Aurore Boucher, L'aléa dans le contrat d'assurance, mémoire de master droit privé, 2009-2010. p. 3. 